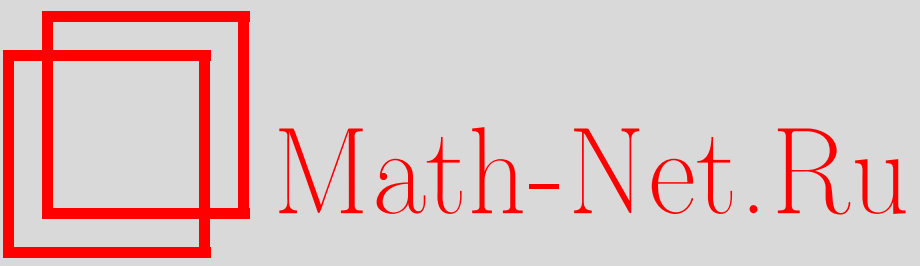

Б. Д. Гельман, Теорема об антиподах и точки совпадения, УМH, 1996, том 51, выпуск 1, 147-148

DOI: https://doi.org/10.4213/rm926

Использование Общероссийского математического портала Math-Net.Ru подразумевает, что вы прочитали и согласны с пользовательским соглашением

http://www.mathnet.ru/rus/agreement

Параметры загрузки:

IP: 3.80 .253 .173

26 апреля 2023 г., 14:02:14 


\title{
ТЕОРЕМА ОБ АНТИПОДАХ И ТОЧКИ СОВПАДЕНИЯ
}

\author{
Б. Д. ГЕЛЬМАН
}

Широко известна роль топологических методов в вопросах разрешимости нелинейных задач. К этому направлению принадлежит и данная работа. Ее особенностью является то, что для доказательства новых теорем существования в ней используются некоторые факты топологической теории многозначных отображений, развитой в последние десятилетия.

Пусть $E^{n}$ - конечномерное линейное пространство. Если $a, b \in E^{n}$, то $[a, b]$ - замкнутый отрезок, соединяющий точки $a$ и $b$.

Пусть $B^{n}$ - замкнутый шар в $E^{n}, S=\partial B^{n}$ - граница шара. Пусть на шаре $B^{n}$ задана непрерывная инволюция $\tau$ без неподвижных точек на $S$, т.е. $\tau: B^{n} \rightarrow B^{n}, \tau^{2}(x)=x$ для любого $x \in B^{n}, \tau(x) \neq x$ для любого $x \in S$.

Пусть $f, g: B^{n} \rightarrow E^{n}$ - непрерывные отображения.

Теорема 1. Отображения $f$ и $g$ удовлетворяют, по крайней мере, одному из двух условий:

(a) отображения $f$ и $g$ имеют точку совпадения $x_{0} \in \operatorname{Int} B^{n}$;

(б) существует точка $x_{*} \in S$ такая, что пересечение

$$
\left[f\left(x_{*}\right): g\left(\tau\left(x_{*}\right)\right)\right] \cap\left[f\left(\tau\left(x_{*}\right)\right): g\left(x_{*}\right)\right] \neq \varnothing .
$$

ДокАЗАТЕльСтво. Если отображения $f$ и $g$ удовлетворяют условию (б), то все доказано. Предположим, что условие (б) не выполняется. Рассмотрим тогда два экземпляра $B_{1}^{n}, B_{2}^{n}$ шара $B^{n}$ и склеим их по границе с помощью тождественного отображения. Полученное множество обозначим $\widehat{B}^{n}$. Очевидно, что множество $\widehat{B}^{n}$ гомеоморфно сфере $S^{n}$. На множестве $\widehat{B}^{n}$ естественно определяется действие непрерьвной инволюции $\widehat{\tau}$ без неподвижных точек следующим образом: на точку $x$ из одного экземпляра шара $B^{n}$ действует инволюция $\tau$, а образ $\tau(x)$ переносится на второй экземпляр шара $B^{n}$.

Рассмотрим многозначное отображение $F: \widehat{B}^{n} \rightarrow E^{n}$, определенное условием

$$
F(x)= \begin{cases}f(x), & \text { если } x \in \operatorname{Int} B_{1}^{n} ; \\ g(\tau(x)), & \text { если } x \in \operatorname{Int} B_{2}^{n} ; \\ {[f(x) ; g(\tau(x))],} & \text { если } x \in S .\end{cases}
$$

Нетрудно убедиться, что $F$ - полунепрерывное сверху многозначное отображение с выпуклыми компактными образами. Для таких отображений справедлива теорема об антиподах (см., например, [2]), т.е. существует такая точка $z_{*} \in \widehat{B}^{n}$, что $F\left(z_{*}\right) \cap F\left(\widehat{\tau}\left(z_{*}\right)\right) \neq \varnothing$. Для точек $z \in S$ пересечение $F(z) \cap F(\widehat{\tau}(z))=\varnothing$ (в силу условия $\left(\right.$ б)). Если $z_{*} \in \operatorname{Int} B_{1}^{n}$, то $F\left(z_{*}\right)=f\left(z_{*}\right)$, $F\left(\widehat{\tau}\left(z_{*}\right)\right)=g\left(\widehat{\tau}\left(z_{*}\right)\right)=g\left(z_{*}\right)$. Следовательно, точка $z_{*}$ является точкой совпадения отображений $f$ и $g$.

СлЕдСТвиЕ. Если для любой точки $x \in S$ пересечение $[f(x) ; g(\tau(x))] \cap[f(\tau(x)) ; g(x)]=\varnothing$, то отобрахсения $f$ u $g$ имеют точку совпадения в Int $B^{n}$.

Полученные утверждения являются новыми даже в случае, когда $f$ является единичным отображением, т.е. когда точка совпадения будет неподвижной точкой $g$.

Пусть $B^{n}$ - замкнутый шар в $E^{n}$ с центром в нуле, а инволюция $\tau: B^{n} \rightarrow B^{n}$ определяется условием $\tau(x)=-x$.

ТЕОрема 2. Если для любой точки $x \in S$ пересечение $[f(x) ; g(-x)] \cap[f(-x), g(x)]=\varnothing$, $m o \operatorname{deg}(f-g, 0) \equiv 1(\bmod 2)$.

ДокаЗАТЕЛЬСтво. Рассмотрим многозначное отображение $F: S \rightarrow E^{n} \backslash 0$, определенное условием

$$
F(x)=\{a-b \mid a \in[f(x) ; g(-x)], b \in[f(-x) ; g(x)]\} .
$$

Очевидно, что отображение $F$ непрерывно по Хаусдорфу и имеет выпуклые компактные образы. Кроме того, $F$ является нечетным, т.е. $F(-x)=-F(x)$ для любого $x \in S$. Тогда (см., например, [1], [2]) $\operatorname{deg}(F, 0) \equiv 1(\bmod 2)$. Так как отображение $f-g$ является сечением $F$, то, в силу свойств степени многозначных отображений, справедливо равенство

$$
\operatorname{deg}(f-g, 0)=\operatorname{deg}(F, 0) \equiv 1 \quad(\bmod 2) .
$$

Рассмотрим обобщение доказанных теорем на случай бесконечномерных пространств.

Пусть $E$ и $V$ - бесконечномерные банаховы пространства, $B$ - шар в $E$ с центром в нуле. 
ОПРЕДЕЛЕНИЕ 1. Флагом подпространств $\left\{E_{n}\right\}$ в пространстве $E$ называется последовательность конечномерных подпространств $E_{n}$, удовлетворяющих следующим условиям: $\operatorname{dim} E_{n}=n, E_{n} \subset E_{n+1}$.

Пусть $f: B \rightarrow V$ - непрерывное отображение, $\left\{E_{n}\right\}$ и $\left\{V_{n}\right\}$ - флаги подпространств в пространствах $E$ и $V$ соответственно.

ОПРЕДЕЛЕнИЕ 2. Отображение $f$ называется допустимым относительно этих флагов, если существует номер $n_{0}$ такой, что множество $f\left(B \cap E_{n}\right) \subset V_{n}$ для любого $n \geqslant n_{0}$.

Например, единичное отображение является допустимым для фолагов $\left\{E_{n}\right\}=\left\{V_{n}\right\}$.

ОПРЕДЕЛЕнИЕ 3. Отображение $f$ назьвается слабо допустимым относительно флагов $\left\{E_{n}\right\}$ и $\left\{V_{n}\right\}$, если для любого $\varepsilon>0$ существует допустимое отображение $f_{\varepsilon}: B \rightarrow V$, удовлетворяющее условию

$$
\inf _{x \in B}\left\|f(x)-f_{\varepsilon}(x)\right\| \leqslant \varepsilon .
$$

Заметим, что если $f$ является допустимым относительно флагов $\left\{E_{n}\right\}$ и $\left\{V_{n}\right\}$, а компактное отображение $k: B \rightarrow V$ таково, что $k(B) \subset \overline{\bigcup_{n} V_{n}}$, то $f+k$ является слабо допустимым относительно тех же флагов.

Теорема 3. Пусть $f: B \rightarrow V$ - собственное непрерывное отображение, слабо допустимое относительно флагов $\left\{E_{n}\right\} u\left\{V_{n}\right\}, g: B \rightarrow V$ - компактное непрерывное отображение. Если 1) $\left.g(B) \subset \bigcup_{n} V_{n}, 2\right)$ существует такое $\varepsilon_{0}>0$, что для любих $x \in \partial B, u \in[f(x) ; g(-x)], v \in[f(-x) ; g(x)]$ выполнено неравенство $\|u-v\| \geqslant \varepsilon_{0}$, то $f u$ $g$ имеют точку совпадения.

ДокАЗАТЕЛЬСтво. Пусть $0<\varepsilon<\varepsilon_{0} / 5, f_{\varepsilon}: B \rightarrow V$ - отображение, сушествуюшее в силу определения 3. Рассмотрим проектор Шаудера $p: \overline{g(B)} \rightarrow V_{n_{1}} \cap \overline{g(B)},\|x-p(x)\|<\varepsilon$ для любого $x \in \overline{g(B)}$.

Без ограничения общности можно сказать, что отображения $f_{\varepsilon}$ и $g_{\varepsilon}=p \circ g$ отображают множество $B_{n_{0}}=B \cap E_{n_{0}}$ в некоторое пространство $V_{n_{0}}$ из флага. Нетрудно доказать, что пересечение $\left[f_{\varepsilon}(x) ; g_{\varepsilon}(-x)\right] \cap\left[f_{\varepsilon}(-x) ; g_{\varepsilon}(x)\right]=\varnothing$ для любого $x \in \partial B$. Отображения $f_{\varepsilon}, g_{\varepsilon}: B_{n_{0}} \rightarrow V_{n_{0}}$, в силу следствия из теоремы 1 , имеют точку совпадения $x_{*}$. Рассмотрим последовательность $\left\{\varepsilon_{n}\right\}, 0<\varepsilon_{n}<\varepsilon_{0} / 5, \varepsilon_{n} \rightarrow 0$. Тогда отображения $f_{n}=f_{\varepsilon_{n}}$ и $g_{n}=g_{\varepsilon_{n}}$ имеют точку совпадения $x_{n}$. Так как последовательность $y_{n}=g\left(x_{n}\right)$ принадлежит компакту $\overline{g(B)}$, то можно считать, что $\left\{y_{n}\right\} \rightarrow y_{*} \in \overline{g(B)}$. Тогда $\left\{f\left(x_{n}\right)\right\} \rightarrow y_{*}$. Пусть $z_{n}=f\left(x_{n}\right)$. Множество $A=y_{*} \cup\left(\bigcup_{n} z_{n}\right)$ является компактом и $x_{n} \in f^{-1}(A)$. В силу собственности отображения $f$, из последовательности $\left\{x_{n}\right\}$ можно выделить сходящуюся подпоследовательность $\left\{x_{n_{i}}\right\},\left\{x_{n_{i}}\right\} \rightarrow x_{*}$. В силу непрерывности отображений $f$ и $g$, заключаем, что точка $x_{*}$ является точкой совпадения этих отображений.

\section{СПИСОК ЛИТЕРАТУРЫ}

[1] Борисович Ю. Г., Гельман Б. Д., Мышкис А. Д., Обуховский В. В. // УМН. 1980. T. 35. № 1. C. 59-126. [2] Górniewicz L. Homological methods in fixed-point theory of multi-valued maps // Rozpr. Mat. 1976. № 129. P. 1-66. 